\title{
X-ray scattering tensor tomography with circular gratings $\odot$
}

\author{
Cite as: Appl. Phys. Lett. 116, 134102 (2020); doi: 10.1063/1.5145361 \\ Submitted: 17 January 2020 - Accepted: 19 March 2020 • \\ Published Online: 1 April 2020

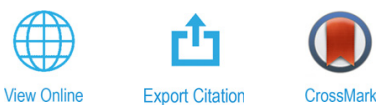

Jisoo Kim, ${ }^{1,2}$ (D) Matias Kagias, ${ }^{2, a)}$ (D) Federica Marone, ${ }^{2}$ (D) and Marco Stampanoni ${ }^{1,2}$ (iD)

\author{
AFFILIATIONS \\ 'Institute of Biomedical Engineering, University and ETH Zurich, 8092 Zurich, Switzerland \\ ${ }^{2}$ Swiss Light Source, Paul Scherrer Institut, 5232 Villigen, Switzerland
}

a) Author to whom correspondence should be addressed: matias.kagias@psi.ch

\begin{abstract}
Three dimensional (3D) information of the microstructure organization of various relevant materials in industry and nature is fundamental to master the understanding of their macroscopic properties. X-ray scattering tensor tomography provides 3D directional information on unresolved microstructures in large volumes, facilitating the investigation of the microstructural organization in statistically large enough sample portions. However, established acquisition protocols such as scanning small angle x-ray scattering and x-ray grating interferometry require long measurement time. In this Letter, a rapid x-ray scattering tensor tomography acquisition method is proposed. It is based on circular gratings, which provide single-shot $2 \mathrm{D}$-omnidirectional information of the sample scattering properties. The main advantage of the presented method is the reduced data acquisition time compared to the existing protocols for x-ray scattering tensor tomography, paving the way toward rapid time-resolved studies.
\end{abstract}

Published under license by AIP Publishing. https://doi.org/10.1063/1.5145361

Macroscopic properties of materials are dictated by their (sub-) microstructural architecture. In biological samples, bone mechanical properties are strongly related to the local orientation of collagen fibers ${ }^{1}$ and the neuronal axon local orientation in the brain determines their structural and functional network. ${ }^{2}$ Similarly in industrial applications, micrometer-thin fibers are utilized to produce reinforced components with superior mechanical properties. The local fiber orientation governs the mechanical and thermal properties of, for instance, injection molded fiber-reinforced materials. ${ }^{3,4}$ Imaging methods providing microscopic spatial resolution are typically employed to investigate the local fiber arrangement. However, these conventional techniques are limited by a strong trade-off between spatial resolution and the examined field of view (FOV). To achieve a spatial resolution on the order of a few micrometers, the investigated FOV is limited to a few $\mathrm{mm}^{2}$. X-ray grating interferometry (XGI) provides information not only on absorption and differential phase contrast but also on small-angle scattering contrast. ${ }^{5-7}$ This small-angle scattering contrast, also known as dark-field contrast, enables the investigation of microscopic features within a macroscopic FOV, effectively decoupling the structure size (correlation length) of interest and the accessible FOV. ${ }^{8-10}$ We simplify the notation of small-angle scattering as scattering for convenience. The potential of x-ray scattering imaging has been demonstrated, for instance, in materials science studies, ${ }^{11}$ biomedical applications, ${ }^{12-14}$ and non-destructive testing for security ${ }^{7}$ and archeology. ${ }^{15}$ In general, scattering is an anisotropic phenomenon and depends on the orientation between the incident beam and the structure. From the measurement of this anisotropic scattering signal, 2D directional scattering contrast can be extracted and anisotropic structures can be investigated. ${ }^{16-19}$ From a collection of projection data with $2 \mathrm{D}$ directional scattering information, taken at different sample angular positions, 3D directional information of microstructures can be tomographically reconstructed. In the x-ray imaging regime, recently developed tensor tomographic methods are based on either $\mathrm{XGI}^{20-22}$ or scanning small-angle x-ray scattering (SAXS). ${ }^{23-25}$ In tensor tomography, a set of parameters (tensor) describing the $3 \mathrm{D}$ directional information of the distribution representing local anisotropic microstructures in each voxel is reconstructed. For this purpose, projection data are acquired not only from different angles around a vertical rotation axis but also around one or more tilted rotation axes along the beam direction. The additional measurements are required to achieve a higher accuracy of the reconstructed microstructure information.

Directional scattering imaging methods based on SAXS require scanning of the sample with a narrow beam (corresponding to the size of a single reconstructed pixel), intrinsically leading to a long acquisition time. XGI, which is a full-field projection imaging technique, 
allows for larger FOVs to be investigated in shorter times. However, in conventional XGI, the directional sensitivity of the measurement depends on the relative orientation of the linear gratings and local anisotropic microstructure. ${ }^{16}$ To expand the directional sensitivity of this technique, a set of measurements is typically required with the sample rotated around an axis parallel to the beam direction.

Instead of a linear grating, a periodic array of multi-circular gratings can be used to capture $2 \mathrm{D}$-omnidirectional $\mathrm{x}$-ray scattering signals within a single projection shot, removing the necessity of rotation of the sample relative to the grating alignment. ${ }^{28}$ Including the additional measurements with tilted rotation axes, the reduction in acquisition time achieved with this single-shot method is significant. Recently, the number of required detector pixels to resolve the fringes in each unit cell has been considerably reduced. As a result, the system autocorrelation length, spatial resolution, and FOV can be more effectively decoupled. ${ }^{29}$ Although speckle-based imaging ${ }^{30}$ could in principle provide similar information, this technique requires high resolution, thus limiting the actual FOV obtained in a single shot. Additionally, for the speckle-based approach, it is still not completely clear how to quantitatively interpret the scattering signal. Here, $x$-ray tensor tomography with circular gratings providing single-shot $2 \mathrm{D}$ omnidirectional scattering sensitivity is presented.

The experimental setup is illustrated in Fig. 1(a). The sample stage is capable of introducing a tilt $(\theta)$ of the rotation $(\varphi)$ axis. The circular unit cell grating has a global period $P$, which is half of the repetition period of the unit cell and a local period $g$, which is the period of the fine circular grating lines within each unit cell as shown in Fig. 1(b). For the parallel beam, at a distance $L=P g / 2 \lambda$ from the sample, where $\lambda$ is the wavelength of the $\mathrm{x}$-rays, the diffraction pattern (fringe) of the gratings is formed [Fig. 1(c)]. The fringe can be directly resolved by a detector placed at this position. The autocorrelation length of the system is $g / 2$. Detailed descriptions of the grating that has been utilized for this work have been recently published by Kagias et al. ${ }^{29}$ The radial line profile going through the center of the circular fringe at an angle $\gamma$ in each unit cell is modeled with two periods of a cosine function. The wavefront of the $\mathrm{x}$-rays is modulated to be rather rough due to scatterings caused by unresolved microstructures, and the visibility of the fringe is reduced as a result. The scattering signal is extracted by the visibility reduction of the local fringe, rather than by analyzing coherent diffraction images. To be more specific, the visibility $V(\gamma)$ is calculated as the ratio of the 2 nd and the 0 th component of the Discrete Fourier Transform (DFT) of the line profile. The 2D directional scattering signal $D(\gamma)$ is then quantified as the visibility reduction $V_{s}(\gamma) / V_{f}(\gamma)$, where $V_{f}(\gamma)$ is the flat image visibility obtained with only gratings in the beam path and $V_{s}(\gamma)$ is the sample image visibility with both gratings and the sample in the beam. The $2 \mathrm{D}$ directional scattering signal for a unit cell $(i, j)$ is therefore expressed as the following equation, where $\tilde{I}(\gamma, N)$ represents the $N$ th component of the DFT of a unit cell fringe in polar coordinates:

$$
D(\gamma)^{(i, j)}=\frac{V_{s}(\gamma)^{(i, j)}}{V_{f}(\gamma)^{(i, j)}}=\frac{\tilde{I}_{s}(\gamma, 2)^{(i, j)} / \tilde{I}_{s}(\gamma, 0)^{(i, j)}}{\tilde{I}_{f}(\gamma, 2)^{(i, j)} / \tilde{I}_{f}(\gamma, 0)^{(i, j)}} .
$$

The 2D directional scattering projection data are collected from different rotation and tilt angles, expanding the dependency of $D$ additionally on $\varphi$ and $\theta$. The notation for all 2D directional scattering data is simplified with a measurement index $m: D(\varphi, \theta, \gamma)^{(i, j)}=D_{m}$.

The unknown $3 \mathrm{D}$ scattering distribution in each voxel is reconstructed using $k=1,2, \ldots, K(K \geq 6)$ different scattering sampling directions. We reconstruct $\mu_{k}(\boldsymbol{x})=\left|\boldsymbol{S}_{k}\right|^{2}$, which represents the squared scattering coefficient along a sampling direction $k$ in voxel $x$, following Malecki et al. ${ }^{20}$ The forward projection model relating $\mu_{k}(\boldsymbol{x})$ to $D_{m}$ can be expressed as a modified form of the Radon transform along the beam path $L_{m}$, which includes an additional scalar term. This scalar term scales the scattering signal by the orientation of the scattering sampling direction $\hat{\boldsymbol{S}}_{k}$ relative to the beam direction vector $\hat{\boldsymbol{B}}_{m}$ and the grating sensitivity direction $\hat{\boldsymbol{G}}_{m}$. Specifically, the forward projection is expressed as Eq. (2), where the scalar term is defined as $v_{k m}:=\left(\left|\hat{\boldsymbol{B}}_{m} \times \hat{\boldsymbol{S}}_{k}\right|\left\langle\hat{\boldsymbol{S}}_{k}, \hat{\boldsymbol{G}}\right\rangle_{m}\right)^{2}$, which can be pre-computed once the acquisition geometry is known. The collection of all measured $p_{m}$,

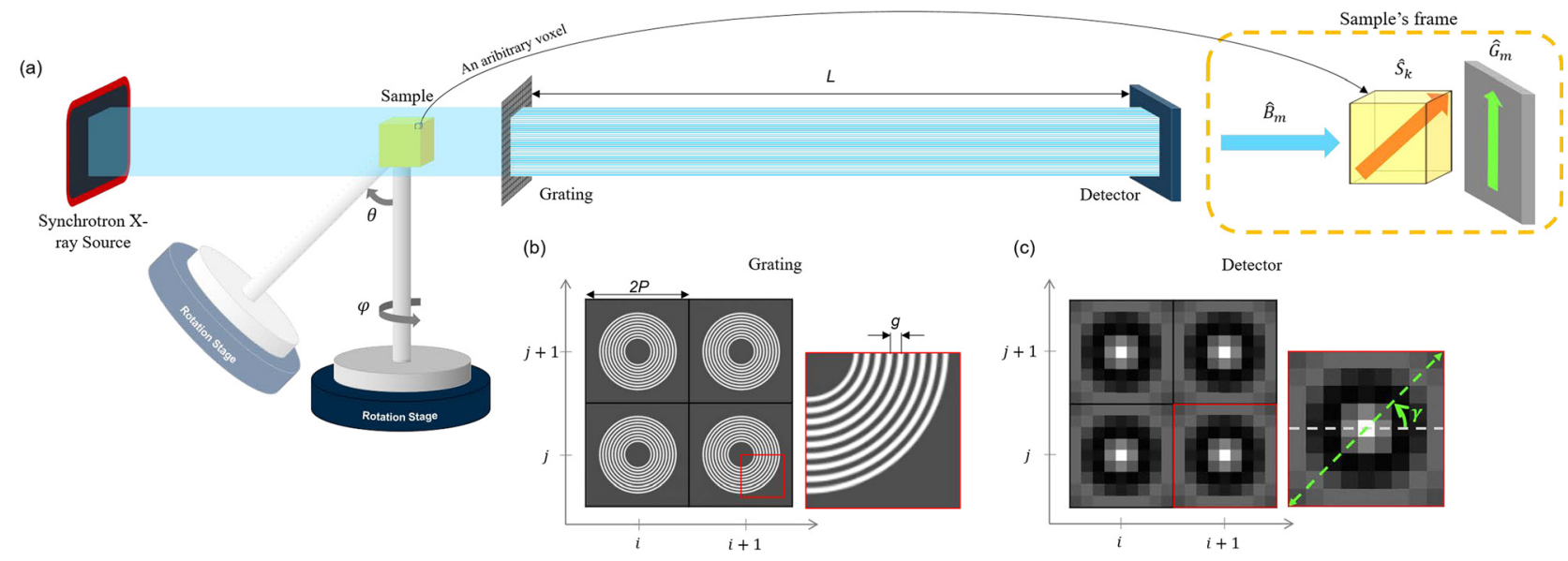

FIG. 1. (a) Side view scheme of the experimental setup. The rotation stage is capable of tilt $(\theta)$ in addition to conventional rotation $(\varphi)$. The x-rays propagate through the sample and (b) the circular phase grating array, reaching the (c) detector plane where the diffraction pattern of each single circular grating is formed in the corresponding square red box called the unit cell. Only $2 \times 2$ unit cells are shown in the schematic. The directional scattering signal is extracted along the green dotted line with an angle $-\pi / 2<\gamma<\pi / 2$ in each unit cell. 
denoted as a projection vector $\boldsymbol{p}$, is expressed as the sum of matrix multiplications of $V_{k W}$ with the vectorized volume $\boldsymbol{\mu}_{k}$, where $V_{k}$ is called the scaling matrix with diagonal elements $v_{k 1}, \ldots, v_{k m}$ and $W$ denotes the geometric system matrix for the discrete beam path integral, leading to a simple system of linear equations ${ }^{21}$

$$
\begin{gathered}
p_{m}=-\ln \left(D_{m}\right)=\sum_{k} v_{k m} \int_{L_{m}} \mu_{k}(\boldsymbol{x}) d \boldsymbol{x}, \\
\boldsymbol{p}=\sum_{k} V_{k} W \boldsymbol{\mu}_{k}=A \boldsymbol{\mu} .
\end{gathered}
$$

The system of linear equations $\boldsymbol{p}=A \boldsymbol{\mu}$ can be solved using a wide range of existing iterative solvers by minimizing $\|\boldsymbol{p}-A \boldsymbol{\mu}\|_{2}^{2}$. In this work, Eq. (3) is solved with the Simultaneous Iterative Reconstruction Technique (SIRT), ${ }^{31}$ and the full equation system is considered in every iterative update. An ellipsoid can accurately approximate the anisotropic scattering signal, if within a voxel there is one dominant structure orientation. $^{20,21,25}$ Thus, principal component analysis can be used to extract the principal axes (eigenvectors) of the ellipsoids modeling the $3 \mathrm{D}$ scattering distribution in each voxel. ${ }^{21}$ The eigenvector with the minimum eigenvalue represents the main orientation of the local microstructure because scattering is typically weakest along the structure main orientation.

The parallel x-ray beam produced at a synchrotron was used, and the energy of the beam was selected to be $17 \mathrm{keV}$ (bandwidth, 2\%-3\%) by a double multi-layer monochromator. With the energy of $17 \mathrm{keV}$, we do not expect any noticeable signal from the Compton scattering or from the fluorescence with carbon and the organic sample used in this experiment. Thus, we have little incoherent contribution from inelastically scattered $\mathrm{x}$-ray photons to the detector plane. The specifically designed $\pi$-shift circular grating array had a unit cell period $P=49.5 \mu \mathrm{m}$ and a fine grating period $g=1.46 \mu \mathrm{m}$. The in-house GigaFRoST $^{32}$ sCMOS detector with a sensor pixel size of $11 \mu \mathrm{m}$ was used with a $300 \mu$ m-thick LuAg:Ce scintillator and a high numerical aperture tandem 1:1 microscope optics. The detector was placed at a distance $L=49.5 \mathrm{~cm}$, and the FOV was $11 \times 5 \mathrm{~mm}^{2}$. The exposure time for each projection was $8 \mathrm{~ms}$, and projection data were acquired with a continuous $360^{\circ}$ rotation of the sample and with tilt angles from $0^{\circ}$ to $45^{\circ}$ with an angular step of $1^{\circ}$. A validation sample was fabricated, where three bundles of carbon fibers with a diameter of approximately $12 \mu \mathrm{m}$ were inserted into a $4 \times 4 \times 4 \mathrm{~mm}^{3}$ PMMA box as shown in Fig. 2(a). The numbers indicate three different carbon fiber bundles with different fiber orientations. A single-shot raw transmission image of this sample is shown in Fig. 2(b), where anisotropic blurring of circular fringes is clearly observable. The fringe in each unit cell was sampled with $9 \times 9$ detector pixel local windows. For comparison and validation of the scattering tensor reconstruction results, the artificial sample was measured using a standard tomographic microscopy setup with a detector pixel size of $6.5 \mu \mathrm{m}$ and $4 \times$ magnification optics. The obtained tomographic slices of the sample are shown in Fig. 2(c). It can be seen that the fibers are more densely packed in bundle 3 than in the other two. In addition, each bundle has local clusters with different fiber densities rather than homogeneously distributed fibers. Also, some of the local clusters within each bundle have slightly deviating orientations compared to the main fiber direction, and the fibers at the edges are bent along the wall.

The tensor reconstruction of the artificial validation sample was performed with 50 SIRT iterations resulting in a volume of (a)
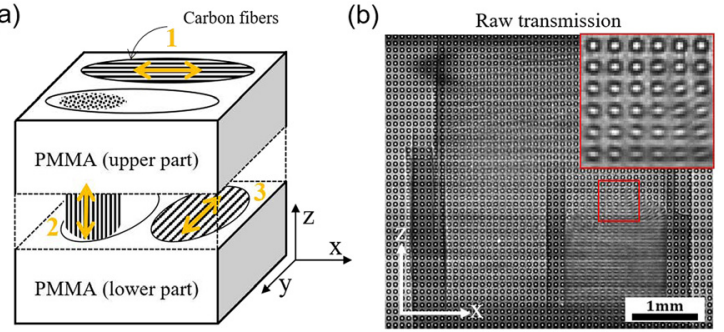

(c)
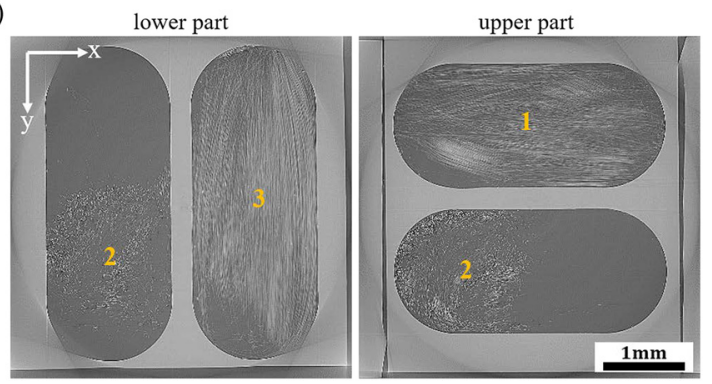

FIG. 2. (a) Schematic illustration of an artificial validation sample, where carbon fiber bundles orthogonal to one another lie in a PMMA box. The orange arrows represent the directions of the fibers in the bundles. (b) Raw transmission image of the validation sample obtained with a single shot at an arbitrary projection angle $\left(\varphi=0^{\circ}, \theta=20^{\circ}\right)$, showing circular fringes. (c) Absorption contrast tomographic slices of the artificial sample reconstructed from projection data measured using a standard x-ray tomographic microscopy approach (pixel size of $1.625 \mu \mathrm{m}$ ). The axial slice images represent the average of 62 consecutive slices, which is equivalent to a single slice of the tensor reconstruction volume at arbitrary locations of the lower and upper PMMA box parts.

$71 \times 71 \times 44$ voxels. For each tilt angle, 50 uniformly distributed angular projections $(50 \times 46=2300$ beam angles $)$ were used. The forward projection operations were performed utilizing the ASTRA Toolbox. ${ }^{33,34}$ The reconstruction time was $\approx 26 \mathrm{~min}$ (single thread implementation, $2.10 \mathrm{GHz}$ Intel Xeon Gold $6130 \mathrm{CPU}$, NVIDIA Quadro P4000 GPU in memory-sharing cluster environment). A 3D visualization of the scattering tensor reconstruction of the artificial sample is shown in Fig. 3. The three bundles shown in Fig. 3(a) are in agreement with the design illustrated in Fig. 2(a) and the tomographic validation in Fig. 2(c). The orientation map is generated using the shortest principal axis of the local scattering ellipsoids, and the average scattering intensity is computed as the mean length of their three principal axes. Local inhomogeneities in the fiber packing, local deviations of the fiber orientation from the main fiber bundle directions, and the bending of fibers along the inner box walls are clearly observable. Sliced orientation maps taken from the lower and upper parts of the artificial sample are shown in Fig. 3(b) and are comparable to Fig. 2(c).

In addition to the artificial validation sample, the end tip of the crown region of a crocodile tooth was also measured and reconstructed with the same experimental conditions and reconstruction algorithm but with 100 SIRT iterations. In this case, we chose a higher number of iterations due to the more complex structure of the sample. Also, a tomographic measurement with an effective pixel size of $0.65 \mu \mathrm{m}$ was performed as a validation of the tensor reconstruction results. The reconstruction results for the tooth sample are shown in Fig. 4. The contour surface images shown in Fig. 4(a) represent the 
(a)

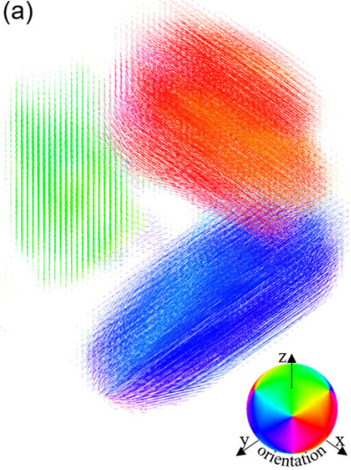

(b)

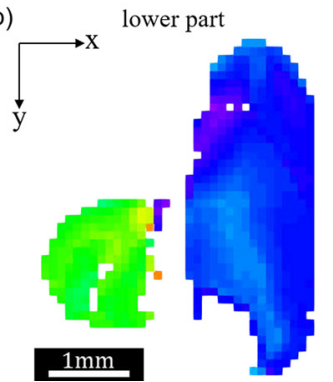

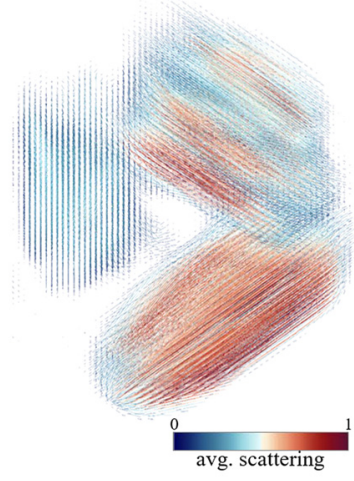

upper part

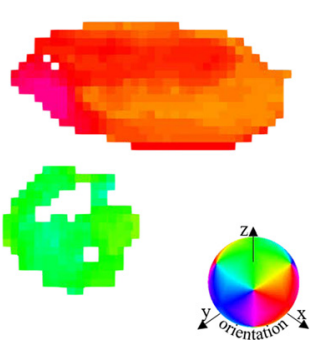

FIG. 3. (a) 3D visualization of the scattering tensor reconstruction of the validation sample from an elevated point-of-view. Each bar represents a voxel, and its orientation corresponds to the fiber direction in each voxel. The two different color maps represent the local fiber orientation (left) and the normalized average scattering intensity (right). (b) Axial slice images from the lower (left) and the upper (right) part of the artificial sample with the color representing the local structure orientation. The color ball is symmetric with respect to the $x-y, x-z$, and $y-z$ planes, and the average scattering color scale is linear.

tooth surface and were generated by thresholding the absorption contrast volumes. The tomographic microscopy results [Fig. 4(b)] show microtubular structures whose diameters are on the order of a few micrometers and a bright shell at the tooth surface representing the enamel layer. We believe that the tubular structures are the dentinal tubules extending from the enamel layer to the pulp where the odontoblasts and fine nerve endings are located. ${ }^{35}$ The tubules are straight along the central axis of the tooth and slightly diverge in the periphery area. The dentinal tubules are, however, not resolved close to the enamel layer in the standard tomographic microscopy reconstructions because their density and diameter decrease as they approach the outer tooth volume. ${ }^{36}$ Nonetheless, these invisible tubular structures generate scattering signals providing information on the local microstructure such as preferred orientation and density. The local structural information in the periphery near the enamel layer is presented in Fig. 4(c) as 3D visualization of the tensor reconstruction of the tooth sample. In agreement with Fig. 4(b), the reconstructed local tubule orientation is aligned vertically in the central area and diverges toward the surface in the periphery area.

In summary, this work demonstrates $\mathrm{x}$-ray scattering tensor tomography with single-shot omnidirectional projections obtained with a circular grating array, without a need for rotation of the grating component or scanning of the sample. An artificial and a biological sample were used to validate the method and show the potential of the technique for realistic specimens. The range of applications can be expanded, e.g., to industrially relevant problems, by transferring the proposed method to x-ray tube setups. The reduced acquisition time opens up new opportunities toward rapid time-resolved $\mathrm{x}$-ray scattering tensor tomographic applications, which could be key for better understanding functional material properties or damage mechanisms, as well as optimizing production processes. Faster acquisition protocols featuring continuous and simultaneous rotation and tilt of the sample leading to spiral trajectories are under investigation. (a)
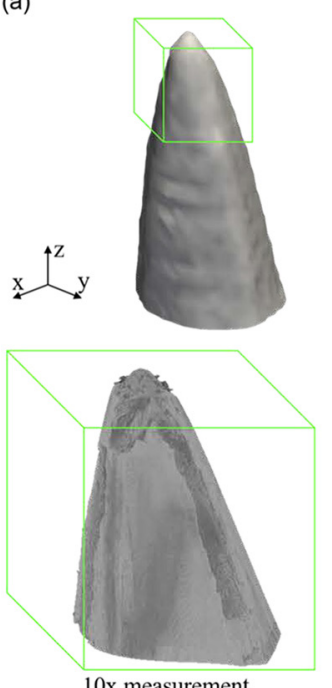

(b)
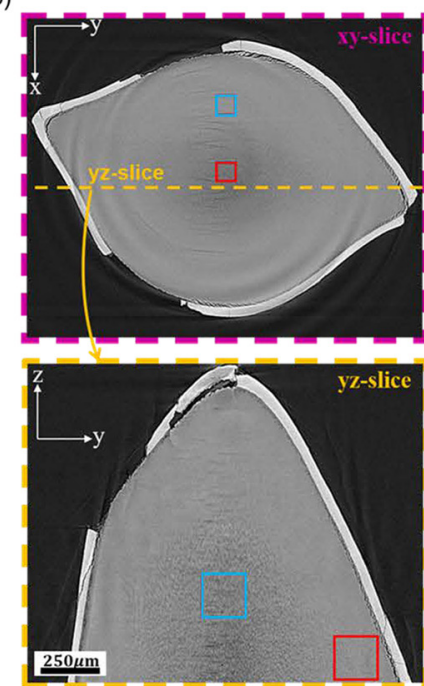
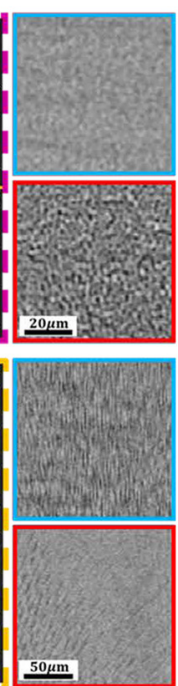

(c)

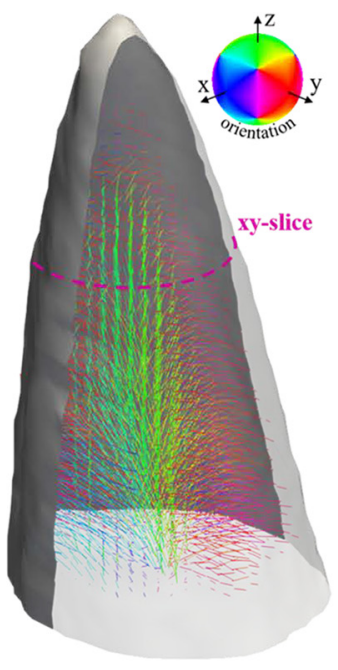

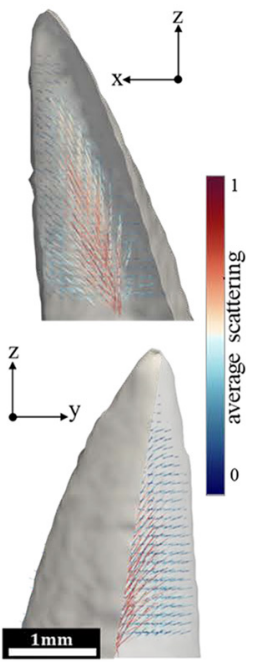

FIG. 4. (a) Contour surface representing the boundary of the tooth, which was generated from the reconstructed volume with absorption contrast. The green box region was independently measured with a 10x magnification optics. (b) Tomographic slices from the $10 \times$ measurement. The $x$-y plane was taken from the bottom of the reconstructed volume, and the $y-z$ plane was taken along the dotted orange line. (c) 3D visualization of the scattering tensor reconstruction of the tooth sample. The color maps represent the local tubule orientation and normalized average scattering. 
This work was part of a project that received funding from the European Union's Horizon 2020 research and innovation programme under Marie Skłodowska-Curie Grant Agreement No. 765604. This work was partially funded by the EUROSTARS INFORMAT E! 11060 project. We would like to thank the Computational Imaging group at CWI for assistance with reconstruction software. The used sample stage was borrowed from the Swiss Light Source cSAXS beamline. The experiments were conducted at the Swiss Light Source TOMCAT beamline, Paul Scherrer Institute, Switzerland.

\section{REFERENCES}

${ }^{1}$ P. Fratzl, "Collagen: Structure and mechanics, an introduction," in Collagen: Structure and Mechanics, edited by P. Fratzl (Springer US, Boston, MA, 2008), pp. 1-13.

${ }^{2}$ S. Jbabdi, S. N. Sotiropoulos, S. N. Haber, D. C. Van Essen, and T. E. Behrens, "Measuring macroscopic brain connections in vivo," Nat. Neurosci. 18, $1546-1555$ (2015)

${ }^{3}$ R. Byron Pipes, R. L. McCullough, and D. G. Taggart, "Behavior of discontinuous fiber composites: Fiber orientation,” Polym. Compos. 3, 34-39 (1982).

${ }^{4} \mathrm{~S}$. T. Chung and T. H. Kwon, "Numerical simulation of fiber orientation in injection molding of short-fiber-reinforced thermoplastics," Polym. Eng. Sci. 35, 604-618 (1995).

${ }^{5}$ A. Momose, S. Kawamoto, I. Koyama, Y. Hamaishi, K. Takai, and Y. Suzuki, "Demonstration of X-ray Talbot interferometry," Jpn. J. Appl. Phys., Part 2 42, L866-L868 (2003).

${ }^{6}$ T. Weitkamp, A. Diaz, C. David, F. Pfeiffer, M. Stampanoni, P. Cloetens, and E. Ziegler, "X-ray phase imaging with a grating interferometer," Opt. Express 13, 6296 (2005).

${ }^{7}$ F. Pfeiffer, M. Bech, O. Bunk, P. Kraft, E. F. Eikenberry, C. Brönnimann, C. Grünzweig, and C. David, "Hard-X-ray dark-field imaging using a grating interferometer," Nat. Mater. 7, 134-137 (2008).

${ }^{8}$ W. Yashiro, Y. Terui, K. Kawabata, and A. Momose, "On the origin of visibility contrast in X-ray Talbot interferometry,” Opt. Express 18, 16890 (2010).

${ }^{9}$ S. K. Lynch, V. Pai, J. Auxier, A. F. Stein, E. E. Bennett, C. K. Kemble, X. Xiao, W.-K. Lee, N. Y. Morgan, and H. Harold Wen, "Interpretation of dark-field contrast and particle-size selectivity in grating interferometers," Appl. Opt. 50, 4310 (2011).

${ }^{10} \mathrm{M}$. Strobl, "General solution for quantitative dark-field contrast imaging with grating interferometers,” Sci. Rep. 4, 7243 (2015).

${ }^{11}$ V. Revol, I. Jerjen, C. Kottler, P. Schütz, R. Kaufmann, T. Lüthi, U. Sennhauser, U. Straumann, and C. Urban, "Sub-pixel porosity revealed by x-ray scatter dark field imaging," J. Appl. Phys. 110, 044912 (2011).

${ }^{12}$ M. Stampanoni, Z. Wang, T. Thüring, C. David, E. Roessl, M. Trippel, R. A. Kubik-Huch, G. Singer, M. K. Hohl, and N. Hauser, "The first analysis and clinical evaluation of native breast tissue using differential phase-contrast mammography," Invest. Radiol. 46, 801-806 (2011).

${ }^{13}$ T. Thüring, R. Guggenberger, H. Alkadhi, J. Hodler, M. Vich, Z. Wang, C. David, and M. Stampanoni, "Human hand radiography using X-ray differential phase contrast combined with dark-field imaging," Skeletal Radiol. 42, 827-835 (2013).

${ }^{14}$ A. Yaroshenko, K. Hellbach, M. Bech, S. Grandl, M. F. Reiser, F. Pfeiffer, and F. G. Meinel, "Grating-based X-ray dark-field imaging: A new paradigm in radiography,” Curr. Radiol. Rep. 2, 57 (2014).

${ }^{15}$ V. Ludwig, M. Seifert, T. Niepold, G. Pelzer, J. Rieger, J. Ziegler, T. Michel, and G. Anton, "Non-destructive testing of archaeological findings by grating-based X-ray phase-contrast and dark-field imaging," J. Imaging 4, 58 (2018).

${ }^{16}$ T. H. Jensen, M. Bech, I. Zanette, T. Weitkamp, C. David, H. Deyhle, S. Rutishauser, E. Reznikova, J. Mohr, R. Feidenhans'l, and F. Pfeiffer, "Directional x-ray dark-field imaging of strongly ordered systems," Phys. Rev. B 82, 214103 (2010).
${ }^{17}$ F. Schaff, A. Malecki, G. Potdevin, E. Eggl, P. B. Noël, T. Baum, E. G. Garcia, J. S. Bauer, and F. Pfeiffer, "Correlation of x-ray vector radiography to bone micro-architecture,” Sci. Rep. 4, 3695 (2015).

${ }^{18}$ F. Prade, F. Schaff, S. Senck, P. Meyer, J. Mohr, J. Kastner, and F. Pfeiffer, "Nondestructive characterization of fiber orientation in short fiber reinforced polymer composites with X-ray vector radiography," NDT\&E Int. 86, 65-72 (2017).

${ }^{19}$ Y. Sharma, S. Bachche, M. Kageyama, M. Kuribayashi, F. Pfeiffer, T. Lasser, and A. Momose, "Trochoidal x-ray vector radiography: Directional dark-field without grating stepping,” Appl. Phys. Lett. 112, 111902 (2018).

${ }^{20}$ A. Malecki, G. Potdevin, T. Biernath, E. Eggl, K. Willer, T. Lasser, J. Maisenbacher, J. Gibmeier, A. Wanner, and F. Pfeiffer, "X-ray tensor tomography," Europhys. Lett. 105, 38002 (2014).

${ }^{21}$ J. Vogel, F. Schaff, A. Fehringer, C. Jud, M. Wieczorek, F. Pfeiffer, and T. Lasser, "Constrained X-ray tensor tomography reconstruction," Opt. Express 23, 15134 (2015).

${ }^{22}$ M. Wieczorek, F. Schaff, F. Pfeiffer, and T. Lasser, “Anisotropic x-ray dark-field tomography: A continuous model and its discretization," Phys. Rev. Lett. 117, 158101 (2016).

${ }^{23}$ M. Liebi, M. Georgiadis, A. Menzel, P. Schneider, J. Kohlbrecher, O. Bunk, and M. Guizar-Sicairos, "Nanostructure surveys of macroscopic specimens by small-angle scattering tensor tomography," Nature 527, 349-352 (2015).

${ }^{24}$ F. Schaff, M. Bech, P. Zaslansky, C. Jud, M. Liebi, M. Guizar-Sicairos, and F. Pfeiffer, "Six-dimensional real and reciprocal space small-angle X-ray scattering tomography," Nature 527, 353-356 (2015).

${ }^{25}$ Z. Gao, M. Guizar-Sicairos, V. Lutz-Bueno, A. Schröter, M. Liebi, M. Rudin, and M. Georgiadis, "High-speed tensor tomography: Iterative reconstruction tensor tomography (IRTT) algorithm,” Acta Crystallogr., Sect. A 75, 223-238 (2019).

${ }^{26}$ Y. Sharma, F. Schaff, M. Wieczorek, F. Pfeiffer, and T. Lasser, "Design of acquisition schemes and setup geometry for anisotropic x-ray dark-field tomography (AXDT)," Sci. Rep. 7, 3195 (2017).

${ }^{27}$ M. Liebi, M. Georgiadis, J. Kohlbrecher, M. Holler, J. Raabe, I. Usov, A. Menzel, P. Schneider, O. Bunk, and M. Guizar-Sicairos, "Small-angle X-ray scattering tensor tomography: Model of the three-dimensional reciprocal-space map, reconstruction algorithm and angular sampling requirements," Acta Crystallogr., Sect. A 74, 12-24 (2018).

${ }^{28}$ M. Kagias, Z. Wang, P. Villanueva-Perez, K. Jefimovs, and M. Stampanoni, "2D-omnidirectional hard-x-ray scattering sensitivity in a single shot," Phys. Rev. Lett. 116, 093902 (2016).

${ }^{29}$ M. Kagias, Z. Wang, M. E. Birkbak, E. Lauridsen, M. Abis, G. Lovric, K. Jefimovs, and M. Stampanoni, "Diffractive small angle X-ray scattering imaging for anisotropic structures," Nat. Commun. 10, 5130 (2019).

${ }^{30}$ T. Zhou, H. Wang, and K. Sawhney, "Single-shot X-ray dark-field imaging with omnidirectional sensitivity using random-pattern wavefront modulator," Appl. Phys. Lett. 113, 091102 (2018).

${ }^{31} \mathrm{P}$. Gilbert, "Iterative methods for the three-dimensional reconstruction of an object from projections," J. Theor. Biol. 36, 105-117 (1972).

${ }^{32}$ R. Mokso, C. M. Schlepütz, G. Theidel, H. Billich, E. Schmid, T. Celcer, G. Mikuljan, L. Sala, F. Marone, N. Schlumpf, and M. Stampanoni, "GigaFRoST: The gigabit fast readout system for tomography," J. Synchrotron Radiat. 24, 1250-1259 (2017).

${ }^{33}$ W. J. Palenstijn, K. J. Batenburg, and J. Sijbers, "Performance improvements for iterative electron tomography reconstruction using graphics processing units (GPUs)," J. Struct. Biol. 176, 250-253 (2011).

${ }^{34}$ W. van Aarle, W. J. Palenstijn, J. Cant, E. Janssens, F. Bleichrodt, A. Dabravolski, J. De Beenhouwer, K. Joost Batenburg, and J. Sijbers, "Fast and flexible X-ray tomography using the ASTRA toolbox," Opt. Express 24, 25129 (2016).

${ }^{35} \mathrm{M}$. Lemmons and D. Beebe, Wiggs's Veterinary Dentistry, 2nd ed. (John Wiley \& Sons, Inc., Hoboken, NJ, USA, 2018), pp. 1-24.

${ }^{36}$ T. L. Lenzi, C. D. A. B. Guglielmi, V. E. Arana-Chavez, and D. P. Raggio, "Tubule density and diameter in coronal dentin from primary and permanent human teeth," Microsc. Microanal. 19, 1445-1449 (2013). 